\title{
Application of the Finite Fourier Sine Transform Method for the Flexural-Torsional Buckling Analysis of Thin-Walled Columns
}

\author{
Mama B.O. ${ }^{1}$, Ike C.C. ${ }^{* 2}$, Nwoji C.U. ${ }^{3}$, Onah H.N. ${ }^{4}$ \\ ${ }^{1}$ Dept of Civil Engineering University of Nigeria, Nsukka, Enugu State, Nigeria. \\ ${ }^{2}$ Dept of Civil Engineering Enugu State University of Science \& Technology, Enugu State, Nigeria. \\ ${ }^{3}$ Dept of Civil Engineering University of Nigeria, Nsukka, Enugu State, Nigeria. \\ ${ }^{4}$ Dept of Civil Engineering University of Nigeria, Nsukka, Enugu State, Nigeria.
}

\begin{abstract}
In this work, the system of three coupled differential equations governing the generalised elastic thinwalled column buckling problem was solved by the finite Fourier sine transform method for the case of pinned ends. The boundary value problem was found to reduce to an algebraic eigenvalue eigenvector problem for which the characteristic buckling equation was found. Two cases were considered. For doubly symmetric sections, it was found that the buckling modes are uncoupled. For monosymmetric sections, it was found that one of the equations is uncoupled, while the other two are coupled. The expressions for the buckling load (eigenvalues) obtained in this study were found to be identical with solutions in literature for the same problem obtained using the method of undetermined parameters.
\end{abstract}

Keywords: finite Fourier sine transform method, monosymmetric cross-sections, eigenvalue problem, characteristic buckling equation, Euler flexural buckling, torsional buckling.

\section{Introduction}

Thin-walled structural elements with cross-sectional shapes such as $工, T, L$ and $[$ with isotropic or anisotropic materials are commonly used as beams, columns and beam columns in engineering applications ranging from buildings, bridges, and aerospace structures. Due to their thin-walled, open crosssections, these elements are always prone to torsion and instabilities [1]. The problem of buckling of thin-walled open-section columns often require complex mathematical analysis, and present problems of considerable magnitude. The modes of failure which form the basis of thin-walled column design are: flexural buckling, local buckling and torsional buckling [2]. Flexural buckling is a sudden deviation of the column from the initial configuration when the critical load is attained. Local buckling will appear as a series of waves in the component parts of the cross-section; while the column remains straight. It is virtually independent of the column length. Torsional buckling occurs when the central part of the column rotates bodily relative to its ends. This occurs even if the ends are free to rotate [2]. These modes of buckling failure do not necessarily occur independently and interaction of the buckling modes frequently occur [2]. Flexural and torsional buckling are often interrelated while flexural and local buckling interact in the post buckling stage.

The buckling behaviour of thin walled columns is very complex and complicated as a result of the interaction effects of compression, flexure and torsional deformations [3]. In structural design, flexural-torsional buckling is an important consideration, particularly for thin-walled members. Flexural-torsional buckling mode involves simultaneous bending and twisting of the cross-section. The cross-section undergoes translational deformation in the two axes of the cross-section, as well as rotational deformation about the shear center. The flexural-torsional buckling problems of thin walled columns has been investigated by Alsayed [4], Timoshenko and Gere [5], Allen and Bulson [6], Chajes [7] and Wang et al [8] and used in the development of the design criteria for steel design. Ike et al [9] have used Galerkin variational method to solve the thin-walled column buckling problem.

\section{Research aim and objectives}

The general aim and objective of this work is to apply the finite Fourier sine transform method to the solution of the generalised flexural-torsional buckling problem of an elastic column with pinned supports at $x=0$ and $x=l$, where $l$ is the length of the column. The specific objectives are:

(i) to use the finite Fourier sine transform method to solve the generalised elastic column buckling problem defined by a system of three coupled differential equations in terms of three unknown displacement functions $v(x), w(x)$ and $\theta(x)$, for the case of pinned support at the ends $x=0$, and $x=l$

\footnotetext{
* Corresponding author
} 
(ii) to show that the generalised elastic column buckling equations reduce to an algebraic eigenvalueeigenvector problem and

(iii) to derive the critical buckling loads for two special cases; namely - columns with doubly symmetric crosssections and columns with singly symmetric cross-sections.

\section{Methodology}

The finite Fourier sine transform was first introduced by Doetsch [13] as a method of integral transformation of boundary value problems. Subsequently, the method has been developed and generalised by several researchers such as Kneitz [14], Strandhagen [15], Roettinger [16] and Brown [17]. The general philosophy behind integral transformations, and the finite Fourier transform method is that they simplify boundary value problems by eliminating partial derivatives with respect to one of the independent variables, hence the transformed equation has one less independent variable.

Definitions: The finite Fourier sine transform $S_{n} u(x)$ of a function $u(x)$ of $x$, is defined as:

$$
S_{n}=S_{n}(u(x))=\int_{0}^{l} u(x) \sin \frac{m \pi x}{l} d x \quad m=1,2,3, \ldots
$$

where $0 \leq x \leq l$

Similarly, the finite Fourier sine transforms of the derivatives are given by

$$
\int_{0}^{l} \frac{\partial u}{\partial x} \sin \frac{m \pi x}{l} d x=u(x=a) \sin m \pi-u(x=0) \sin 0-\frac{m \pi}{l} \int_{0}^{l} u \cos \frac{m \pi x}{l} d x
$$

where $\int_{0}^{l} u \cos \frac{m \pi x}{l} d x$ is the finite Fourier cosine transform of $u(x)$

Also,

$$
\int_{0}^{l} \frac{\partial^{2} u}{\partial x^{2}} \sin \frac{m \pi x}{l} d x=-\alpha_{m}\left((-1)^{m} u(x=l)-u(x=0)\right)-\alpha_{m}^{2} \int_{0}^{l} u(x) \sin \alpha_{m} x d x
$$

where $\alpha_{m}=m \pi / l$

$$
\begin{gathered}
\int_{0}^{l} \frac{\partial^{3} u}{\partial x^{3}} \sin \alpha_{m} x d x=-\alpha_{m}\left(\left.(-1)^{m} \frac{\partial u}{\partial x}\right|_{x=l}-\left.\frac{\partial w}{\partial x}\right|_{x=0}\right)+\alpha_{m}^{3} \int_{0}^{l} u \cos \alpha_{m} x d x \\
\int_{0}^{l} \frac{\partial^{4} u}{\partial x^{4}} \sin \alpha_{m} x d x=-\alpha^{4} \int_{0}^{l} u \sin \alpha_{m} x d x+\alpha_{m}^{3}\left(u(x=l)(-1)^{m}-u(x=0)\right) \\
-\alpha_{m}\left((-1)^{m} \frac{\partial^{2} w}{\partial x^{2}}(x=l)-\frac{\partial^{2} w}{\partial x^{2}}(x=0)\right)
\end{gathered}
$$

\section{Theoretical Framework - flexural-torsional governing equations of buckling elastic columns}

Consider an elastic column of length $l$, whose longitudinal axis is defined by the $x$ coordinate, and the plane of the cross-section is defined by the $y z$ Cartesian coordinate plane. The system of governing differential equations that describe the flexural-torsional buckling behaviour of a generalized elastic column under an axial compressive load $N$, acting through the centroid of the cross-section if the moments due to the transverse loads are zero, the applied torque vanishes; and the load is applied such that the bi-moment is zero are given by the following $[10,11,12]$ :

$$
\begin{aligned}
& E I_{z z} \frac{d^{4} v}{d x^{4}}+N_{x} \frac{d^{2} v}{d x^{2}}+N_{x} e_{z} \frac{d^{2} \theta}{d x^{2}}=0 \\
& E I_{y y} \frac{d^{4} w}{d x^{4}}+N_{x} \frac{d^{2} w}{d x^{2}}-N_{x} e_{y} \frac{d^{2} \theta}{d x^{2}}=0
\end{aligned}
$$




$$
E C_{w} \frac{d^{4} \theta}{d x^{4}}-\left(G J-\frac{I_{E} N_{x}}{A}\right) \frac{d^{2} \theta}{d x^{2}}+e_{z} N_{x} \frac{d^{2} v}{d x^{2}}-e_{y} N_{x} \frac{d^{2} w}{d x^{2}}=0
$$

where $v(x), w(x)$ and $\theta(x)$ are the displacements

$E=$ Young's modulus of elasticity

$G=$ shear modulus or modulus of rigidity

$C_{w}=$ warping constant

$I_{z z}=$ moment of inertia about the $z$ axis

$I_{y y}=$ moment of inertia about the $y$ axis

$e_{z}=$ coordinate of the shear centre

$e_{y}=$ coordinate of the shear centre

$N_{x}=$ load in the $x$ - direction

$I_{E}=$ polar moment of inertia about the shear centre

$J=$ St Venant torsional stiffness of the section

$$
I_{E}=I_{y y}+I_{z z}+\left(e_{y}^{2}+e_{z}^{2}\right) A
$$

$A=$ area of cross-section.

The governing equations are a system of three differential equations in terms of the three displacements $v(x)$, $w(x)$ and $\theta(x)$.

\section{Application of the finite Fourier Sine Transform Method to the flexural-torsional buckling problem}

We seek to apply the finite Fourier Sine transform method to find solutions to the system of governing differential equations for a column of length $l$ with pinned ends at $x=0$ and $x=l$. The unknown functions in the governing system of differential equations are the three displacement functions, namely $v(x), w(x)$ and $\theta(x)$; and we seek a solution to the elastic column buckling problem for the case of pinned-pinned end supports (i.e.at $x=0$ and $x=l$ ). The boundary conditions for pinned-pinned supports at $x=0$, and $x=l$ are:

$$
\begin{aligned}
& v(x=0)=v(x=l)=0 \\
& w(x=0)=w(x=l)=0 \\
& \theta(x=0)=\theta(x=l)=0 \\
& v^{\prime \prime}(x=0)=v^{\prime \prime}(x=l)=0 \\
& w^{\prime \prime}(x=0)=w^{\prime \prime}(x=l)=0 \\
& \theta^{\prime \prime}(x=0)=\theta^{\prime \prime}(x=l)=0
\end{aligned}
$$

where $v^{\prime \prime}=\frac{d^{2} v}{d x^{2}}, w^{\prime \prime}=\frac{d^{2} w}{d x^{2}}$ and $\theta^{\prime \prime}=\frac{d^{2} \theta}{d x^{2}}$ and the primes denote differentiation with respect to the coordinate variable $x$.

The boundary conditions make the application of the finite Fourier Sine transform method viable for the problem for the case of pinned supports at $x=0$, and $x=l$. Hence, applying the finite Fourier sine transforms to the system of three differential equations, we obtain:

$$
\begin{gathered}
\int_{0}^{l}\left(E I_{z z} v^{i v}(x)+N_{x} v^{\prime \prime}(x)+N_{x} e_{z} \theta^{\prime \prime}(x)\right) \sin \frac{m \pi x}{l} d x=0 \\
\int_{0}^{l}\left(E I_{y y} w^{i v}(x)+N_{x} w^{\prime \prime}(x)-N_{x} e_{y} \theta^{\prime \prime}(x)\right) \sin \frac{m \pi x}{l} d x=0 \\
\int_{0}^{l}\left(E C_{w} \theta^{i v}(x)-\left(G J-\frac{I_{E} N_{x}}{A}\right) \theta^{\prime \prime}(x)+N_{x} e_{z} v^{\prime \prime}(x)-N_{x} e_{y} w^{\prime \prime}(x)\right) \sin \frac{m \pi x}{l} d x=0
\end{gathered}
$$

Simplifying,

$E I_{z z} \int_{0}^{l} \frac{d^{4} v(x)}{d x^{4}} \sin \frac{m \pi x}{l} d x+N_{x} \int_{0}^{l} \frac{d^{2} v(x)}{d x^{2}} \sin \frac{m \pi x}{l} d x+N_{x} e_{z} \int_{0}^{l} \frac{d^{2} \theta(x)}{d x^{2}} \sin \frac{m \pi x}{l} d x=0$ 


$$
\begin{gathered}
E I_{y y} \int_{0}^{l} \frac{d^{4} w}{d x^{4}} \sin \frac{m \pi x}{l} d x+N_{x} \int_{0}^{l} \frac{d^{2} w}{d x^{2}} \sin \frac{m \pi x}{l} d x-N_{x} e_{y} \int_{0}^{l} \frac{d^{2} \theta}{d x^{2}} \sin \frac{m \pi x}{l} d x=0 \\
E C_{w} \int_{0}^{l} \frac{d^{4} \theta}{d x^{4}} \sin \frac{m \pi x}{l} d x-\left(G J-\frac{I_{E} N_{x}}{A}\right) \int_{0}^{l} \frac{d^{2} \theta}{d x^{2}} \sin \frac{m \pi x}{l} d x \\
+e_{z} N_{x} \int_{0}^{l} \frac{d^{2} v}{d x^{2}} \sin \frac{m \pi x}{l} d x-e_{y} N_{x} \int_{0}^{l} \frac{d^{2} w}{d x^{2}} \sin \frac{m \pi x}{l} d x=0
\end{gathered}
$$

Using integration by parts, we find:

$$
\begin{aligned}
& \int_{0}^{l} \frac{d^{4} v(x)}{d x^{4}} \sin \frac{m \pi x}{l} d x=\left(\frac{m \pi}{l}\right)^{4} \int_{0}^{l} v(x) \sin \frac{m \pi x}{l} d x=\left(\frac{m \pi}{l}\right)^{4} V(m, x) \\
& \int_{0}^{l} \frac{d^{2} v}{d x^{2}} \sin \frac{m \pi x}{l} d x=-\left(\frac{m \pi}{l}\right)^{2} \int_{0}^{l} v(x) \sin \frac{m \pi x}{l} d x=-\left(\frac{m \pi}{l}\right)^{2} V(m, x) \\
& \int_{0}^{l} \frac{d^{2} \theta}{d x^{2}} \sin \frac{m \pi x}{l} d x=-\left(\frac{m \pi}{l}\right)^{2} \int_{0}^{l} \theta(x) \sin \frac{m \pi x}{l} d x=-\left(\frac{m \pi}{l}\right)^{2} \theta(m, x) \\
& \int_{0}^{l} \frac{d^{4} w}{d x^{4}} \sin \frac{m \pi x}{l} d x=\left(\frac{m \pi}{l}\right)^{4} \int_{0}^{l} w(x) \sin \frac{m \pi x}{l} d x=\left(\frac{m \pi}{l}\right)^{4} W(m, x) \\
& \int_{0}^{l} \frac{d^{2} w}{d x^{2}} \sin \frac{m \pi x}{l} d x=-\left(\frac{m \pi}{l}\right)^{2} \int_{0}^{l} w(x) \sin \frac{m \pi x}{l} d x=-\left(\frac{m \pi}{l}\right)^{2} W(m, x)
\end{aligned}
$$

where

$$
\begin{aligned}
& V(m, x)=\int_{0}^{l} v(x) \sin \frac{m \pi x}{l} d x \\
& W(m, x)=\int_{0}^{l} w(x) \sin \frac{m \pi x}{l} d x \\
& \theta(m, x)=\int_{0}^{l} \theta(x) \sin \frac{m \pi x}{l} d x
\end{aligned}
$$

and $V(m, x)$ is the finite Fourier Sine transform of $v(x), W(m, x)$ is the finite Fourier Sine transform of $w(x)$ and $\theta(m, x)$ is the finite Fourier Sine transform of $\theta(x)$.

The system of governing differential equations become:

$$
\begin{gathered}
E I_{z z}\left(\frac{m \pi}{l}\right)^{4} V(m, x)-N_{x}\left(\frac{m \pi}{l}\right)^{2} V(m, x)-N_{x} e_{z}\left(\frac{m \pi}{l}\right)^{2} \theta(m, x)=0 \\
E I_{y y}\left(\frac{m \pi}{l}\right)^{4} W(m, x)-N_{x}\left(\frac{m \pi}{l}\right)^{2} W(m, x)+N_{x} e_{y}\left(\frac{m \pi}{l}\right)^{2} \theta(m, x)=0 \\
E C_{w}\left(\frac{m \pi}{l}\right)^{4} \theta(m, x)+\left(G J-\frac{I_{E} N_{x}}{A}\right)\left(\frac{m \pi}{l}\right)^{2} \theta(m, x) \\
-N_{x} e_{z}\left(\frac{m \pi}{l}\right)^{2} V(m, x)+N_{x} e_{y}\left(\frac{m \pi}{l}\right)^{2} W(m, x)=0
\end{gathered}
$$


We divide through by $\left(\frac{m \pi}{l}\right)^{2}$ to obtain

$$
\begin{aligned}
& E I_{z z}\left(\frac{m \pi}{l}\right)^{2} V(m, x)-N_{x} V(m, x)-N_{x} e_{z} \theta(m, x)=0 \\
& \left(E I_{z z}\left(\frac{m \pi}{l}\right)^{2}-N_{x}\right) V(m, x)-N_{x} e_{z} \theta(m, x)=0 \\
& \left(E I_{y y}\left(\frac{m \pi}{l}\right)^{2}-N_{x}\right) W(m, x)+N_{x} e_{y} \theta(m, x)=0 \\
& \left(E C_{w}\left(\frac{m \pi}{l}\right)^{2}+\left(G J-\frac{I_{E} N_{x}}{A}\right)\right) \theta(m, x)-N_{x} e_{z} V(m, x)+N_{x} e_{y} W(m, x)=0
\end{aligned}
$$

This is a system of homogeneous algebraic equations in terms of $V(m, x), W(m, x)$ and $\theta(m, x)$ and can be written in matrix form as:

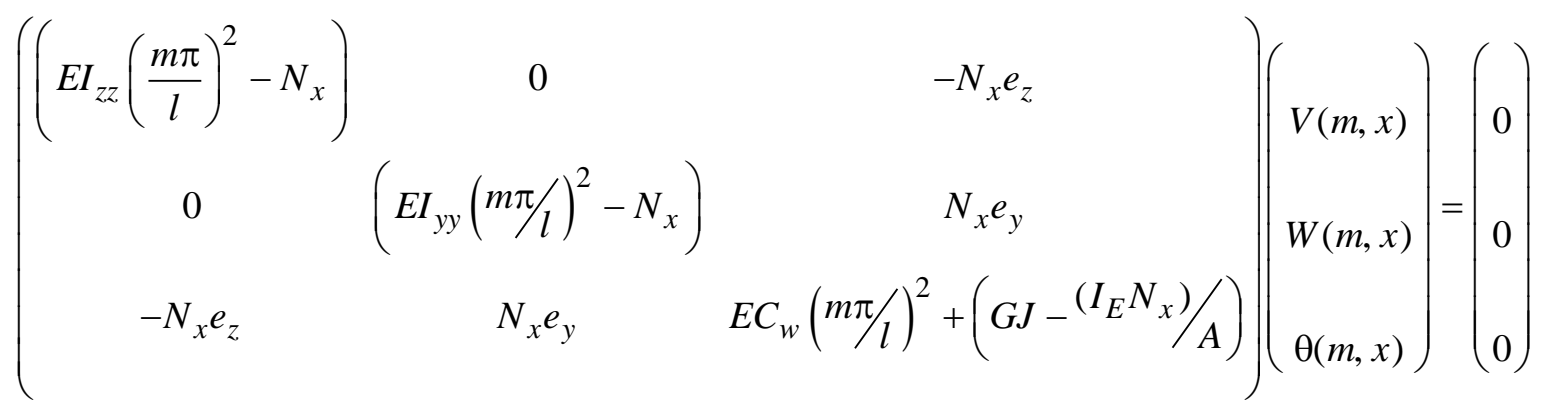

For nontrivial solutions, the determinant of the coefficient matrix must vanish, and the requirement for the vanishing of the coefficient matrix yields the characteristic equation or stability equation for the problem. Hence, the stability equation is given by:

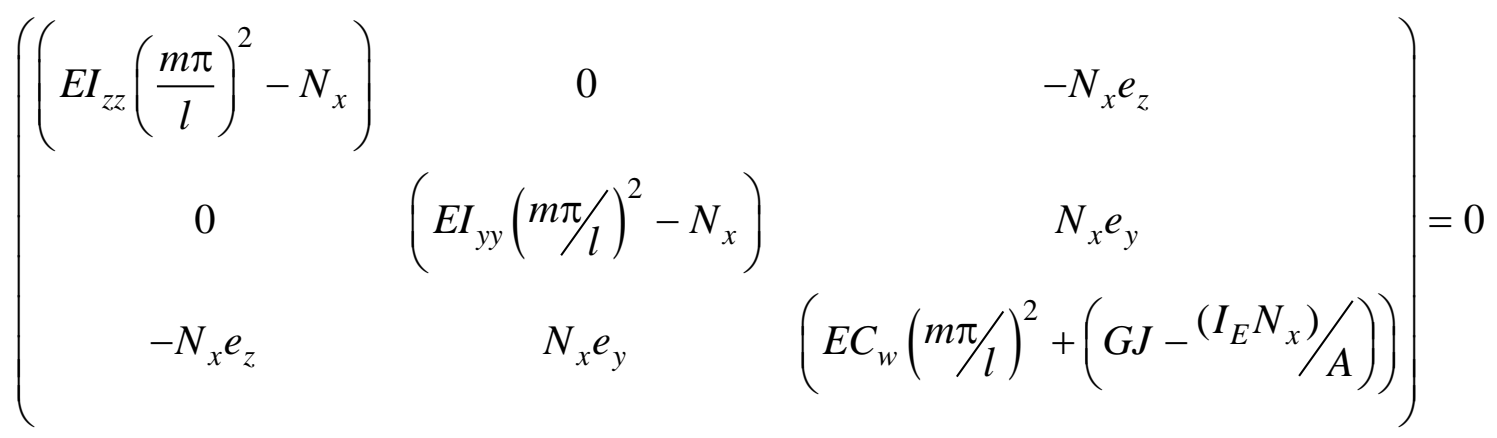

The characteristic buckling equation for the flexural torsional problem of elastic column with pinned ends at $x=$ 0 , and $x=l$ is obtained by the expansion of the determinantal equation (Equation (33)), and finding the roots or the zeros of the resulting polynomial in $N_{x}$. Two particular cases of this problem, which can be considered as simplifications of the general flexural-torsional buckling problem are considered.

Case 1: The cross section of the elastic column is doubly symmetric about the $y$ and $z$ coordinate axes. Some typical examples of doubly symmetric cross sections are symmetric $I$ sections, and crucifix sections. For doubly symmetric cross sections, $e_{y}=e_{z}=0$ and the characteristic buckling equation simplifies to Equation (34). 


$$
\left(\begin{array}{ccc}
\left(E I_{z z}\left(\frac{m \pi}{l}\right)^{2}-N_{x}\right) & 0 & 0 \\
0 & \left(E I_{y y}(m \pi / l)^{2}-N_{x}\right) & 0 \\
0 & 0 & \left(E C_{w}(m \pi / l)^{2}+G J-\left(I_{E} N_{x}\right) / A\right.
\end{array}\right)=0
$$

Expansion of the characteristic stability equation yields the factorized form of the polynomial in $N_{x}$ :

$$
\left(E I_{z z}\left(\frac{m \pi}{l}\right)^{2}-N_{x}\right)\left(E I_{y y}\left(\frac{m \pi}{l}\right)^{2}-N_{x}\right)\left(E C_{w}\left(\frac{m \pi}{l}\right)^{2}+G J-\frac{I_{E} N_{x}}{A}\right)=0
$$

The buckling equations are found to be uncoupled as follows:

$$
\begin{aligned}
& E I_{z z}\left(\frac{m \pi}{l}\right)^{2}-N_{x}=0 \\
& E I_{y y}\left(\frac{m \pi}{l}\right)^{2}-N_{x}=0 \\
& E C_{w}\left(\frac{m \pi}{l}\right)^{2}+G J-\frac{I_{E} N_{x}}{A}=0
\end{aligned}
$$

The three roots of the characteristic buckling equation are:

$$
\begin{aligned}
& N_{x}=E I_{z z}\left(\frac{m \pi}{l}\right)^{2}=P_{E_{z z}} \\
& N_{x}=E I_{y y}\left(\frac{m \pi}{l}\right)^{2}=P_{E_{y y}} \\
& N_{x}=\frac{A}{I_{E}}\left(E C_{w}\left(\frac{m \pi}{l}\right)^{2}+G J\right)=P_{t}
\end{aligned}
$$

where $P_{E_{z z}}$ is the Euler load for flexural buckling about the $z z$ axis, $P_{E_{y y}}$ is the Euler load for flexural buckling about the $y y$ axis and $P_{t}$ is the buckling load in torsional (twist) buckling. The stress $\left(\sigma_{x x}^{t}\right)$ in torsional buckling is

$$
\sigma_{x x}^{t}=-\frac{1}{I_{p}}\left(E C_{w}\left(\frac{m \pi}{l}\right)^{2}+G J\right)=\frac{P_{t}}{A}
$$

where $I_{p}=I_{E}$ for doubly symmetric cross-section.

Case 2: In this case, the cross section is singly symmetric with respect to the coordinate axes. A typical example of singly symmetric cross-section is the channel section. If the $z$ axis is the axis of symmetry, then $e_{y}=0$ and $e_{z} \neq 0$. The characteristic buckling equation simplifies to become: 


$$
\left(\begin{array}{ccc}
\left(E I_{z z}\left(\frac{m \pi}{l}\right)^{2}-N_{x}\right) & 0 & -N_{x} e_{z} \\
0 & \left(E I_{y y}(m \pi / l)^{2}-N_{x}\right) & 0 \\
-N_{x} e_{z} & 0 & \left(E C_{w}(m \pi / l)^{2}+G J-\left(I_{E} N_{x}\right) / A\right.
\end{array}\right)=0
$$

Expansion of the equation yields:

$$
\left(E I_{y y}\left(\frac{m \pi}{l}\right)^{2}-N_{x}\right)\left\{\left(E I_{z z}\left(\frac{m \pi}{l}\right)^{2}-N_{x}\right)\left(E C_{w}\left(\frac{m \pi}{l}\right)^{2}+G J-\frac{I_{E} N_{x}}{A}\right)-\left(N_{x} e_{z}\right)^{2}\right\}=0
$$

Solving, we have

$$
\begin{aligned}
& E I_{y y}\left(\frac{m \pi}{l}\right)^{2}-N_{x}=0 \\
& \left(E I_{z z}\left(\frac{m \pi}{l}\right)^{2}-N_{x}\right)\left(E C_{w}\left(\frac{m \pi}{l}\right)^{2}+G J-\frac{I_{E} N_{x}}{A}\right)-\left(N_{x} e_{z}\right)^{2}=0
\end{aligned}
$$

The roots are:

$$
\begin{aligned}
& N_{x}=E I_{y y}\left(\frac{m \pi}{l}\right)^{2}=P_{E_{y y}} \\
& N_{x}=\frac{\left(P_{t}+P_{E_{z z}}\right) \pm \sqrt{\left[\left(P_{t}+P_{E_{z z}}\right)^{2}-4\left(1-\frac{A e_{z}^{2}}{I_{E}}\right) P_{E_{z z}} P_{t}\right]}}{2\left(1-\frac{e_{z}^{2} A}{I_{E}}\right)} \\
& N_{x}=\frac{1}{2 \beta}\left[\left(P_{t}+P_{E_{z z}}\right) \pm \sqrt{\left(P_{t}+P_{E_{z z}}\right)^{2}-4 \beta P_{E_{z z}} P_{t}}\right]
\end{aligned}
$$

where $P_{E_{z z}}=E I_{z z}\left(\frac{m \pi}{l}\right)^{2}$

$$
\begin{aligned}
& \beta=1-\frac{A e_{z}^{2}}{I_{E}} \\
& P_{t}=\frac{A}{I_{E}}\left(E C_{w}\left(\frac{m \pi}{l}\right)^{2}+G J\right)
\end{aligned}
$$

The expression for $N_{x}$ given by Equation (48) represents the buckling load for the coupled flexural toritional buckling mode. A negative sign in Equation (48) would yield smaller values for $N_{x}$.

Hence, 


$$
\begin{aligned}
& N_{x}=\frac{\left(P_{E_{z z}}+P_{t}\right)-\sqrt{\left[\left(P_{t}+P_{E_{z z}}\right)^{2}-4\left(1-\frac{A e_{z}^{2}}{I_{E}}\right) P_{t} P_{E_{z z}}\right]}}{2\left(1-\frac{A e_{z}^{2}}{I_{E}}\right)} \\
& N_{x}=\frac{1}{2 \beta}\left[\left(P_{t}+P_{E_{z z}}\right)-\sqrt{\left(P_{t}+P_{E_{z z}}\right)^{2}-4 \beta P_{E_{z z}} P_{t}}\right]
\end{aligned}
$$

Critical buckling loads are obtained when $m=1$ in the Equations (47) and (48). Critical buckling loads are thus obtained as:

$$
N_{x}=E I_{y y} \frac{\pi^{2}}{l^{2}}=P_{E_{y y}}^{c}
$$

and

$$
\begin{aligned}
& N_{x}=\frac{\left(P_{t}^{c}+P_{E_{z z}}^{c}\right)-\sqrt{\left(P_{t}^{c}+P_{E_{z z}}^{c}\right)^{2}-4\left(1-\frac{A e_{z}^{2}}{I_{E}}\right) P_{E_{z z}}^{c} P_{t}^{c}}}{2\left(1-\frac{e_{z}^{2} A}{I_{E}}\right)} \\
& N_{x}=\frac{1}{2 \beta}\left[\left(P_{t}^{c}+P_{E_{z z}}^{t}\right)-\sqrt{\left(P_{t}^{c}+P_{E_{z z}}^{c}\right)^{2}-4 \beta P_{E_{z z}}^{c} P_{t}^{c}}\right]
\end{aligned}
$$

where the superscript $c$ is used to denote critical.

The critical buckling stress $\sigma_{x x_{c r}}^{f T}$ for the coupled flexural-torsional buckling mode is obtained as:

$$
\begin{aligned}
& \sigma_{x x_{c r}}^{f T}=\frac{\left(\sigma_{x x_{c r}}^{E}+\sigma_{x x_{c r}}^{T}\right)-\sqrt{\left[\left(\sigma_{x x_{c r}}^{E}+\sigma_{x x_{c r}}^{T}\right)^{2}-4\left(1-\frac{A e_{z}^{2}}{I_{E}}\right) \sigma_{x x_{c r}}^{E} \sigma_{x x_{c r}}^{T}\right]}}{2\left(1-\frac{A e_{z}^{2}}{I_{E}}\right)} \\
& \sigma_{x x_{c r}}^{f T}=\frac{1}{2 \beta}\left[\left(\sigma_{x x_{c r}}^{E}+\sigma_{x x_{c r}}^{T}\right)-\sqrt{\left(\sigma_{x x_{c r}}^{E}+\sigma_{x x_{c r}}^{T}\right)^{2}-4 \beta \sigma_{x x_{c r}}^{E} \sigma_{x x_{c r}}^{T}}\right]
\end{aligned}
$$

$$
\begin{aligned}
\text { where } \sigma_{x x_{c r}}^{E}=\frac{E \pi^{2}}{\left(l / r_{y}\right)^{2}} \\
\sigma_{x x_{c r}}^{T}=\frac{E}{I_{E}}\left(C_{w} \frac{\pi^{2}}{l^{2}}+\frac{J}{2(1+\mu)}\right)
\end{aligned}
$$

\section{Discussion of Results}

This work has successfully applied the finite Fourier Sine transform method to the flexural-torsional buckling analysis of thin-walled open section columns with both ends simply supported. The finite sine transform was applied to the governing equations which were a system of three coupled differential equations in terms of the three displacement functions $v(x), w(x)$ and $\theta(x)$. After simplification, the system of transformed equations reduced to a system of homogeneous algebraic equations in terms of the three transformed 
displacement functions $V(m, x), W(m, x)$ and $\theta(m, x)$ as presented in Equation (32). The buckling or characteristic equation is obtained from the requirement of vanishing of the determinant of the matrix of coefficients and obtained as Equation (33). The zeros of the resulting polynomial equation in $N_{x}$ would yield the buckling loads of the column.

Two cases of the general buckling problem were considered: namely - case of doubly symmetric crosssections and singly symmetric cross-sections. For doubly symmetric cross-sections, the shear center coincide with the centroid of the cross-section, yielding $e_{y}=e_{z}=0$. The system of governing differential equations become uncoupled, resulting in buckling modes, that are uncoupled. The characteristic buckling equation for doubly symmetric sections found as equation (35); is uncoupled, with three roots representing the eigenvalues of the buckling problem. The three roots are the Euler flexural buckling load in the $z$ axis, the Euler flexural buckling load in the $y$ axis, and the load in torsional (twist) buckling. The critical buckling load is the lowest value of the three buckling loads, and determines how the column with doubly symmetrical cross section will fail. The second case considered columns with monosymmetric cross-sections, where $z z$ axis is the axis of symmetry, $e_{y}=0$, and from the system of governing differential equations, Equation (7) is uncoupled from Equations (6) and (8). The characteristic buckling equation was found in this case as Equation (45) a polynomial equation in $N_{x}$ with one flexural buckling mode uncoupled and the other flexural buckling mode coupled with the torsional buckling mode. Thus such monosymmetric columns can fail by Euler flexural buckling mode in the yy direction and torsional-flexural buckling mode. The zeros of the polynomial (equivalent to the eigenvalues) yield the values of the buckling load. The polynomial would yield three values of the buckling load namely Euler flexural buckling load in the yy direction and two coupled torsional-flexural buckling loads given by Equation (48). Evidently, Equation (50) would give lower values of the torsional-flexural buckling load; and the buckling mode would be governed by $N_{x}$ in Equation (52) or the Euler flexural buckling load in the $y$-direction, whichever one is smaller. The expressions for the buckling loads obtained by finite Fourier Sine transform method agree excellently with solutions by Det [11] and Wang et al [8].

\section{Conclusion}

For this study, the following conclusions can be adduced:

(i) For doubly symmetric thin-walled columns, the system of governing differential equations are uncoupled resulting in buckling modes and buckling loads that are uncoupled.

(ii) For monosymmetric thin walled columns, with $z z$ axis being the axis of symmetry, flexural buckling mode in the $y$ direction is uncoupled while the flexural buckling mode in the $z$ direction is coupled with torsional buckling.

(iii) For thin walled coluns without symmetry about any axis, the three buckling modes are coupled and the two flexural buckling modes interact with torsional buckling mode.

The finite Fourier Sine transform method has been shown to be an effective tool for the analysis of thin-walled columns with pinned ends. The advantage of the method is that no apriori assumption about buckling modes was necessary to solve the system of differential equations. The method yielded exact solutions.

\section{References}

[1] Zhu Shuai: Elastic Flexural Torsional Buckling Analysis of Doubly Symmetrical Web Tapered Beams. MSc Thesis, University of Pittsburgh, Oct 2009, 208pp.

[2] Howlett J.H.: An investigation into the structural behaviour of thin-walled aluminum alloy welded battened struts. MSc Thesis, University of Durham, July 1972, available at Durham E-Thesis online http//ethesis.dur.ac.uk/10293.

[3] Trahair N.S.: Torsion Equations for lateral buckling. Research Report R964, July 2016, School of Engineering, The University of Sydney.

[4] Alsayed S.H.: Inelastic behaviour of single angle columns. PhD Thesis, The University of Arizona, University Microfilms International. http//ndl.handler.net/10150/184041. 1987

[5] Timoshenko S.P. and Gere J.M.: Theory of Elastic Stability. McGraw Hill Kogakusha Ltd, New York, 1961.

[6] Allen H.G. and Bulson P.S.: Background to Buckling. McGraw Hill Book Co., 1980.

[7] Chajes A.: Principles of Structural Stability Theory. Prentice Hall, New Jersey, 1974.

[8] Wang C.M., Wang C.Y. and Reddy J.N.: Exact Solution for Buckling of Structural Members. CRC Series in Computational Mechanics and Applied Mechanics, CRC Press, USA, 2005.

[9] Ike C.C., Nwoji C.U., Ikwueze E.U. and Ofondu I.O.: Solution of the Generalised Elastic Column Buckling Problem by the Galerkin Variational Method. International Journal of Research in Applied Science and Engineering Technology (IJRASET), Volume 5 Issue 1, pp. 468-475, January 2017.

[10] Torsion in Structural Design. http//people.virginia.edu/ttb/torsion.pdf

[11] Det N.V.: Buckling Strength Analysis of Bars and Frames, and Spherical Shells Classification Notes No 30.1 . Norway, April 2004.

[12] Trahair N.S.: Flexural-Torsional Buckling of Structures. CRC Press Tokyo, 1993.

[13] Doetsch G.: Integration von Differentialgleichungen vermittels der endlichen Fourier transformation Math. Ann, 112, pp 52-68, 1935 .

[14] Kneitz K.: Lösung von Rondwert probleme bei systemem gewöhulicher Differentialgleichungen vermittels der endlichen Fourier transformation, Math. Zeit, 44, pp 266-291, 1938

[15] Strandhagen A.G.: Use of sine transform for non-simply supported beams Quart. Appl. Math 1, pp.346-348, 1944. 
Application of the Finite Fourier Sine Transform Method for the Flexural-Torsional Buckling ..

[16] Roettinger I.: An operational approach to the solution of boundary value problems by generalised Fourier series. Bull American Math. Soc. 51 p.67, 1947.

[17] Brown H.K.: Resolution of temperature problems by use of finite Fourier transformations. Bull. American Math. Soc., 50, pp. 376$385,1944$. 